\title{
Using ISS Telescopes for Electromagnetic Follow-up of Gravitational Wave Detections of NS-NS and NS-BH Mergers
}

\author{
J. Camp · S. Barthelmy · L. Blackburn · K. Carpenter · N. Gehrels · J. Kanner · \\ F. E. Marshall · J. L. Racusin · T. Sakamoto
}

Received: date / Accepted: date

\begin{abstract}
The International Space Station offers a unique platform for rapid and inexpensive deployment of space telescopes. A scientific opportunity of great potential later this decade is the use of telescopes for the electromagnetic followup of ground-based gravitational wave detections of neutron star and black hole mergers. We describe this possibility for OpTIIX, an ISS technology demonstration of a $1.5 \mathrm{~m}$ diffraction limited optical telescope assembled in space, and ISS-Lobster, a wide-field imaging X-ray telescope now under study as a potential NASA mission. Both telescopes will be mounted on pointing platforms, allowing rapid positioning to the source of a gravitational wave event. Electromagnetic follow-up rates of several per year appear likely, offering a wealth of complementary science on the mergers of black holes and neutron stars.
\end{abstract}

\section{Introduction}

The ISS has recently completed its construction activities and is now moving into its period of utilization, providing space payloads with a long-term, stable platform 11 Its large solar arrays and Ku-band antennas provide the ISS with power and data capabilities which are capable of supporting multiple payloads. The result is that scientific payloads can be attached to the ISS without needing their own power supply or data downlink capability, and with reduced requirements on the attitude control system, thus significantly reducing the cost and time required to fly an experiment in space. Also, since payloads are launched to the station on independently scheduled rockets, they do not need to fund their own dedicated rocket, further reducing the cost of the mission.

Astrophysics Science Division, NASA Goddard Space Flight Center, Greenbelt, MD

${ }^{1}$ http://www.nasa.gov/mission_pages/station/research/benefits
One such payload is the Optical Testbed and Integration on ISS Experiment (OpTIIX), a mission to demonstrate the capability of robotically assembling a $1.5 \mathrm{~m}$ diffraction limited optical telescope in space. The key long-term goal of this technology is to enable the robotic assembly of a future $10 \mathrm{~m}+$ size telescope capable of characterizing extrasolar terrestrial planets and searching them for signs of life. OpTIIX also has a number of potential science applications, including monitoring of outer solar system planetary atmospheres, stellar population studies of nearby star-forming regions, and fast followup of astrophysical transient events. OpTIIX has completed a Preliminary Design Review and is now seeking funding for the construction phase, with a possible operation start in 2017.

Another potential payload, now in the conceptual study phase to address an upcoming NASA Mission of Opportunity, is ISS-Lobster, a wide-field imaging X-ray telescope. Based on glancing incidence focusing of 0.3-5 keV X-rays, ISS-Lobster is intended to provide a simultaneous wide field of view, high spatial resolution, and high sensitivity, to advance the field of time-domain X-ray astronomy. Its scientific goals include the study of tidal disruptions of stars by supermassive black holes, the observation of supernova shock breakouts, gamma-ray bursts, and the long-term variability of active galactic nuclei.

Both OpTIIX and ISS-Lobster are planned for mounting on platforms which will be in nearly continuous communication with ground-based alert networks through the ISS data link, allowing the fast pointing of the telescopes in response to an astronomical transient. This suggests the possibility of their use in an extremely important scientific scenario around the middle of this decade, the electromagnetic follow-up of ground-based gravitational wave detections. Gravitational waves are produced by the interaction of extremely dense and massive objects including black holes (BH) and neutron stars (NS). Mergers of these compact ob- 
jects also produce electromagnetic radiation: NS-NS and/or NS-BH mergers are likely to be the source of short GammaRay Bursts, a very bright transient source of gamma, optical and X-ray signals, and also the source of a predicted longlived optical afterglow known as a kilonova. The observation of BH and NS mergers is likely later this decade with the availability of a network of gravitational wave detectors; the coincident observation of a compact object merger event in the electromagnetic bands would provide a rich complement to the science of the merger, and crucially, would also help to verify the event itself.

In this article we give an overview of the OpTIIX and ISS-Lobster designs. We then describe the network of planned gravitational wave detectors, and then discuss the possibilities for electromagnetic follow-up observations with the ISS telescopes.

\section{ISS telescopes}

\subsection{OpTIIX}

The OpTIIX (Optical Testbed and Integration on ISS Experiment) instrument (Postman, M. et al. 2012) consists of six modules that will be launched separately and assembled robotically on the ISS, including six deformable primary mirror segments. An overview of the experiment is as follows. The modules as shown in figure 1 are: the Telescope Core Module, including the avionics, star trackers to determine the telescope position, the wavefront sensing and control unit used to focus the telescope, and the imaging camera; the Gimbal Module to provide three degree of freedom pointing; the Secondary Tower Module which contains the secondary and tertiary mirrors and coarse steering mirror; and three Mirror Segment Modules, each of which contain two primary mirror segments and their actuators, along with laser metrology components.

The modules will be assembled on the ISS with the use of the Special Purpose Dexterous Manipulator (SPDM), the ISS robot arm. All of the telescope components handled by SPDM will contain the necessary interfaces and attachment mechanisms. The SPDM has an articulating body, an assortment of tools, cameras and sensors, and a pair of sevenjointed arms to allow efficient assembly of OpTIIX. The ISS location of OpTIIX has not yet been determined.

The assembled telescope can be described as follows. The primary mirror, comprised of six hexagonal segments of $50 \mathrm{~cm}$ diameter, has the shape of a conic asphere, and has a diameter of $1.45 \mathrm{~m}$. The primary shape is adjusted by means of six rigid-body actuators, and 90 shape actuators, attached to each mirror segment backing structure. The actuators provide primary figure control, as well as high precision alignment of the segments to the telescope. Laser metrology, enabled with a frequency stabilized laser source

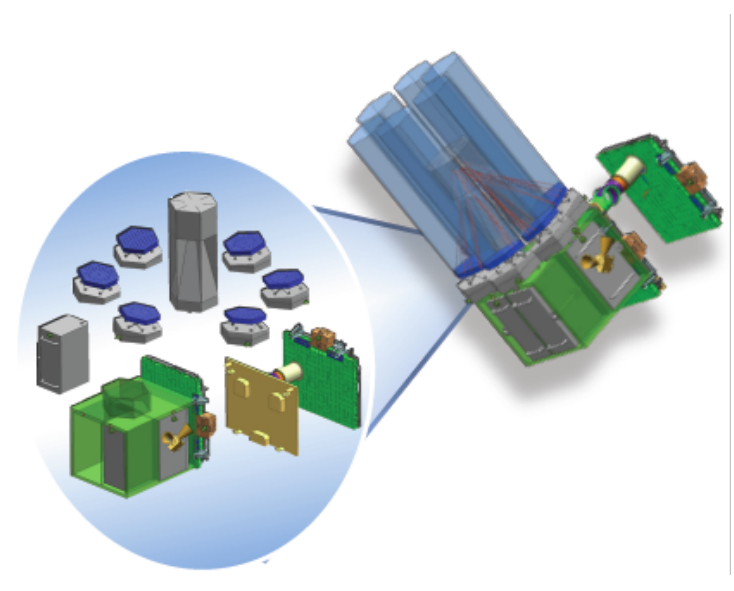

Fig. 1 Exploded view of OpTIIX

(Numata, K. et al. 2010; Leonhardt, V. \& Camp, J. 2006) and beam launchers on the primary and fiducials on the secondary, provides the measurement of the distance and alignment between the primary and secondary (Zhao, F. et al. 2006). The telescope is focused with image-based wavefront sensing and control algorithms. The focus process proceeds in three stages: Shack-Hartmann sensing for the segments initial alignment, dispersed fringe sensing for segment co-phasing, and phase-retrieval sensing for the final wavefront adjustment to achieve diffraction-limited performance. An imaging camera is used to obtain the diffractionlimited images, and will include a filter wheel with eight positions, including three wide-band and three narrow-band optical filters. The final OpTIIX performance specifications are: diffraction limited performance with a Strehl ratio of $80 \%$ at $600 \mathrm{~nm}$, signal-to-noise (SNR) of 5 for a star of AB magnitude 22 or fainter in a total exposure time of $20 \mathrm{~min}$, and field of view of $\sim 3$ arc min square.

Jitter noise on the ISS from anthropogenic sources as well as the expression of ISS structural modes is attenuated with the use of a pointing platform which also serves to position the telescope at a given sky location. This is a key technical challenge for OpTIIX since the full telescope performance corresponds to a sub arc sec pointing requirement. The current pointing control design includes three loops: star trackers and gyro input to the gimbal, coarse steering mirror control using data from the gyro, and tertiary mirror control using feedback from the fine guidance sensor. Trade studies are ongoing.

Once assembled and operational, OpTIIX will be tasked with the acquisition of at least 50 color images of diffractionlimited astronomical targets for education and public outreach (EPO) use. A set of science observations will also be undertaken, to the extent that time permits. 


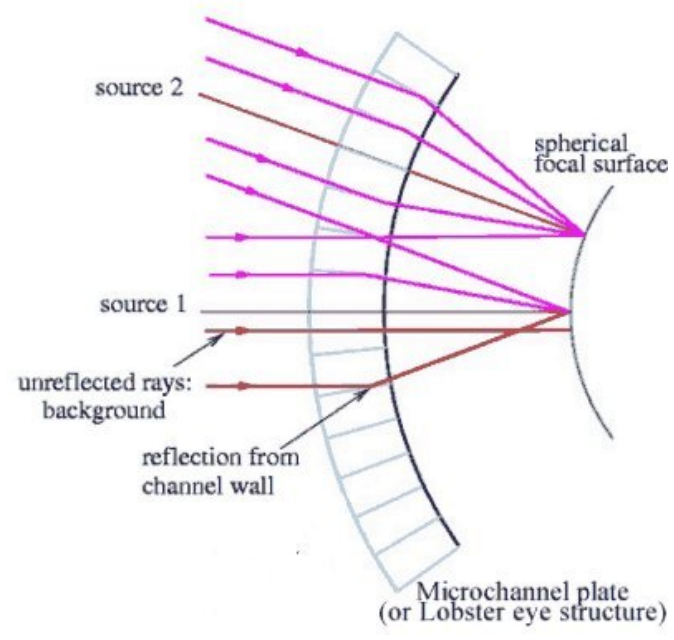

(a) Lobster focusing optic

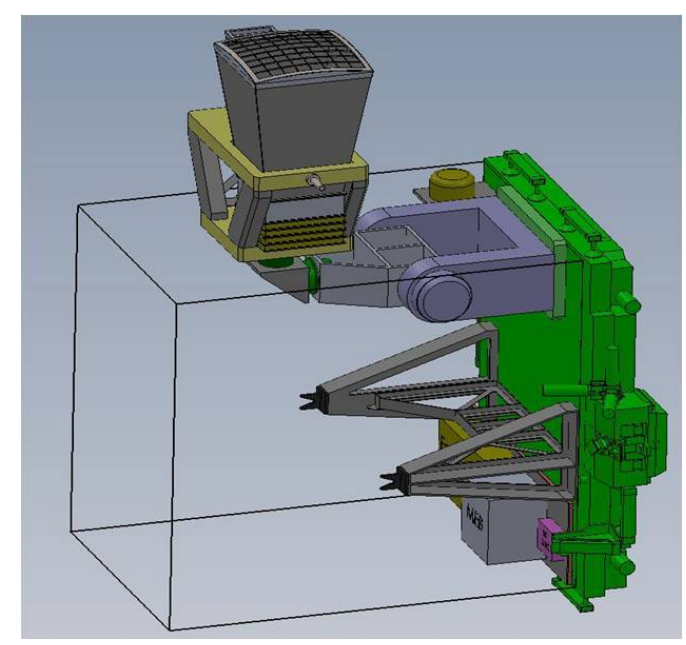

(b) ISS-Lobster instrument on 3-axis gimbal

Fig. 2 ISS-Lobster elements

\subsection{ISS-Lobster}

ISS-Lobster is a wide-field X-ray telescope now under study at Goddard Space Flight Center, planned to be situated on the ISS Express Logistics Carrier. It consists of two instruments, the soft X-ray (0.3-5 keV) Wide Field Imager (WFI), and the hard X-ray (10-1000 keV) Gamma-ray Transient Monitor (GTM). It simultaneously provides wide field of view (FOV), high position resolution, and high sensitivity. These characteristics enable the search for transient X-ray events.

Figure 2(a) shows the principle of operation, which is similar to that employed (in the optical band) by the namesake crustacean (Black, J. et al.2003). The WFI X-ray optic consists of an array of square-packed channels, molded into a spherical shape. The long axes of the channels intersect a distance $\mathrm{R}$ along the axis of symmetry. X-rays undergo- ing odd numbers of grazing reflection are brought to a focus a distance $\mathrm{R} / 2$ along the symmetry axis. This gives rise to a cruciform point-spread function consisting of a crossshaped pattern (caused by X-ray reflections an odd number of times in one plane and an even number of times in the other) with a central focus, and a uniform background (caused by an even number of reflections in both planes). The ratio of the components is about $25 \%$ in the focus, $50 \%$ in the cross-arms, and $25 \%$ diffuse background.

ISS-Lobster WFI will consist of a microchannel plate array with $75 \mathrm{~cm}$ radius of curvature, with an area of $40 \mathrm{~cm} \times$ $40 \mathrm{~cm}$. The focal plane detector will be a CCD camera, with image resolution of about $250 \mu \mathrm{m}$ (FWHM), and energy resolution better than $100 \mathrm{eV}$. These parameters give rise to a wide field X-ray telescope with FOV of $30^{\circ}$ x $30^{\circ}$, an angular resolution of 8 arcmin FWHM and 1 arcmin localization accuracy, and a sensitivity of $1.3 \times 10^{-11} \mathrm{erg} /\left(\mathrm{cm}^{2} \mathrm{sec}\right)$ in $2000 \mathrm{sec}$ for an energy range of 0.3 to $5 \mathrm{keV}$. The focusing capability gives ISS-Lobster over a factor of 30 more sensitivity than previous wide-field X-ray telescopes - e.g. the BAT detector on Swift (Barthelmy, S. et al.2005) and the GSC detector on MAXI (Matsuoka, M. et al.2010).

The WFI module will be deployed on a pointing platform to remove the ISS jitter, and to allow fast positioning (Figure 2(b) This will require a control system consisting of a three-axis gimbal and star tracker. Initial studies have shown that pointing at the level of 1 arcmin is achievable.

The WFI FOV of $\sim 2 \%$ of the sky will enable the detection of gamma-ray bursts ( $\gtrsim 0.3 /$ day), supernova shock breakouts $(\sim 1-2 / \mathrm{yr})$, and tidal disruptions of stars by supermassive black holes ( $\gtrsim 14 / \mathrm{yr}$ ). Many other sources will be available for study, including active galactic nuclei, stellar flares, and neutron star bursts.

ISS-Lobster GTM consists of a single Sodium Iodide (NaI) scintillation detector identical to those comprising the Fermi-GBM. It will provide high time resolution data on transient sources over an energy range of $10-1000 \mathrm{keV}$. While it will provide no source localization within its FOV, the simultaneous gamma-ray trigger with a GW event will confirm the astrophysical origin of the GW source, and allow for the possible verification of sub-threshold GW triggers.

\section{LIGO and the ground-based gravitational-wave network}

Gravitational radiation arises in regions of strong and dynamical gravitational fields from astronomical sources, including very dense and massive objects such as black holes and neutron stars. Its character describes the nature of gravity in the extreme, testing the predictions of Einsteins theory of gravity and providing information about its sources unobtainable through other means. In contrast to the electromagnetic waves of conventional astronomy, which arise 
from the incoherent superposition of emission from the acceleration of individual electric charges, gravitational waves result from coherent, bulk motions of matter. Also, because gravitational waves interact only weakly with matter they are able to penetrate the very densely concentrated matter that produces them. The direct detection and study of gravitational waves has the potential to revolutionize our understanding of the universe.

A gravitational wave produces a differential strain (ratio of change in distance to distance between two points) in space-time along orthogonal directions, and can be observed through the relative timing of the passage of light waves along these directions. Because gravity is a very weak force, the strain expected at the earth from the gravitational waves of the most likely astrophysical sources is very small, of order $10^{-21}$.

A number of detectors around the world have been built, or are being constructed, to search for gravitational waves. The general design is shown in figure 3 and is based on the principle of laser interferometry (Abbott, B. et al. 2004). A frequency stabilized laser injects light into an interferometer beam splitter, where the light is split and directed downstream through a light storage arm to test mass end mirrors, and then reflected back and recombined at the beamsplitter. The interferometer is operated so that destructive interference of the recombined light occurs; the photodiode monitoring the output light of the interferometer is then highly sensitive to any differential motion of the mirrors which disturbs the exact cancellation of the light. A very high level of displacement sensitivity, of order $10^{-18} \mathrm{~m} \mathrm{rms}$, is achieved through careful attention to the frequency and amplitude stability of the laser light, the losses of the interferometer optics, the seismic isolation system which decouples the interferometer from motions of the earth, and the suspension system which stabilizes and positions the optics. Kilometer scale detectors are required to enable sufficient strain sensitivity for gravitational wave detection.

Gravitational wave signals anticipated by these groundbased detectors include: continuous waves from non-axisymmetr pulsars, stochastic backgrounds of sources from the early universe, burst signals from a non-axisymmetric supernova explosion or other unmodeled event, and signals from the inspiral and merger of a compact binary system with a black hole and/or neutron star. The most promising of these sources for the first detection is the NS-NS or NS-BH merger, which becomes likely with a detector strain sensitivity of $10^{-22}$ rms, integrated over the detector frequency range of $10 \mathrm{~Hz}$ to $2000 \mathrm{~Hz}$.

The gravitational wave network of detectors includes the Laser Interferometer Gravitational Wave Observatory (LIGO) (Abbott, B. et al. 2009), with $4 \mathrm{~km}$ detectors in Hanford, Washington (Figure 4(a)) and Livingston, Louisiana; the 3 km Virgo (Accadia, T. 2011) observatory in Pisa, Italy; the

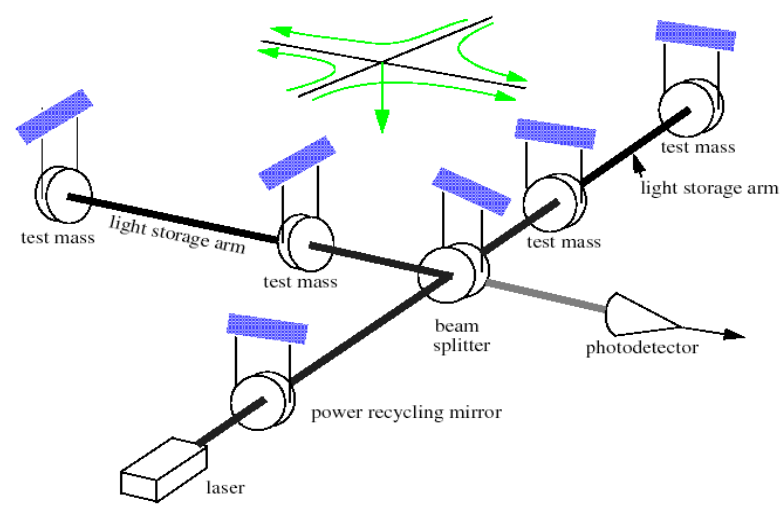

Fig. 3 Gravitational wave interferometric detector schematic

$600 \mathrm{~m}$ GEO (Grote, H. et al. 2010) interferometer in Germany; the $3 \mathrm{~km}$ KAGRA (Somiya, K. et al. 2012) detector under construction in Japan; and a $4 \mathrm{~km}$ detector recently approved for construction in India (Sathyaprakash, B. et al. 2012). LIGO and Virgo completed in 2010 a one year period of observation, and both projects are undergoing an upgrade to their detectors ${ }^{2}$ (Harry, G. et al. 2010). Full sensitivity for these detectors is expected for LIGO and Virgo around 2018, for KAGRA around 2020, and for the India detector in 2022. At full sensitivity LIGO will have a detection range for a NS-NS binary merger of $200 \mathrm{Mpc}$, and for a NS-BH merger of $400 \mathrm{Mpc}$. The detection rate for these sources is uncertain to a factor of 10 in both directions, but the highest probability is 40/yr and 30/yr, respectively Abadie, J. et al. 2010).

As more detectors become operational in the network the result will be a higher detection sensitivity, as well as a better sky location of sources: for three detectors, the sky error box for the binary NS merger will be $\sim 100 \mathrm{deg}^{2}$ at a network SNR of 10, but with four and five detectors the error becomes $20 \mathrm{deg}^{2}$ and $5 \mathrm{deg}^{2}$, respectively (Fairhurst, S. 2011). For the time frame of the deployment of the ISS relescopes, 2016-2017, the gravitational wave network will consist of the three detectors from LIGO and Virgo.

\section{Electromagnetic Follow-up of Gravitational Wave Detections with OpTIIX and ISS-Lobster}

\subsection{Electromagnetic counterpart science}

The detection of electromagnetic counterparts to gravitational wave signals from these NS-NS and NS-BH mergers with OpTIIX and/or ISS-Lobster will powerfully enhance the sci-

\begin{tabular}{cccc}
\hline 2 Advanced $\quad$ Virgo documentation & and & notes \\
https://wwwcascina.virgo.infn.it/advirgo/docs.html & &
\end{tabular}




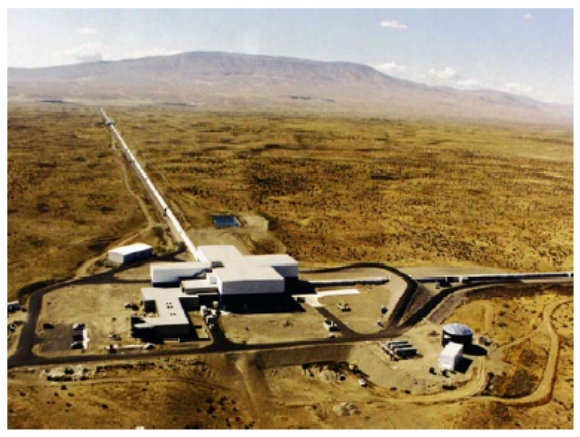

(a) Aerial view of LIGO site at Hanford, WA

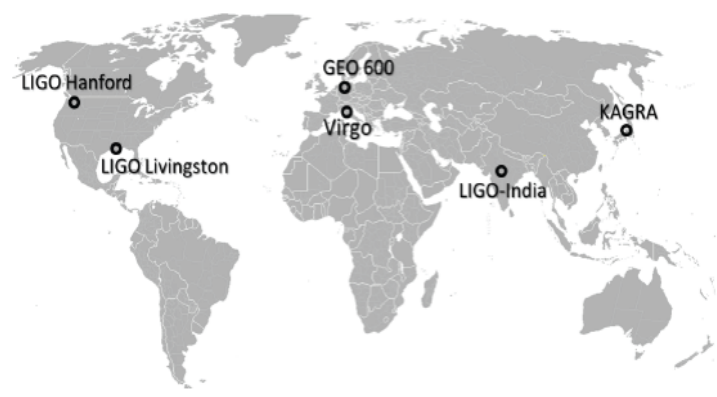

(b) World network of GW detectors

Fig. 4 Current and future ground-based interferometric GW detectors

ence of the compact merger event. In brief, the detection of an EM counterpart will:

- Locate the galaxy of the source progenitor. The position resolution of OpTIIX and ISS-Lobster is at the level of 1 arcsec and 1 arcmin, respectively, both easily high enough to locate the progenitor galaxy for an event within the LIGO detection range of $400 \mathrm{Mpc}$.

- Determine the source redshift. With the galaxy location determined, ground based spectroscopic telescopes can follow-up the detection and determine the host redshift. When combined with the source distance that can be extracted from the gravitational waveform, this could lead to a more precise local determination of $\mathrm{H}_{0}$, the Hubble constant (Dalal, N. et al.2006).

- Deliver information on source beaming, and examine the afterglow energetics to probe the progenitor and its gas environment.

- Test predictions of General Relativity, including the speed and polarizations of gravitational waves.

- Lift degeneracies associated with the binary source parameters (Hughes, S. \& Holz, D. 2003). Extraction of key binary source information such as luminosity distance and inclination is limited by the poor sky localization of the Gravitational Wave (GW) network. The precise sky localization enabled by the EM counterpart signal will assist in the analysis of the gravitational waveform, allowing significantly higher precision in the extraction of source parameters.

- Raise the confidence level of a GW detection. The coincident measurement of an EM signal with a GW detection, both spatially and temporally, will greatly aid in reducing false detections. This can allow the lowering of the GW detection threshold, extending the range of the measurement.

- Determine the engine of the short Gamma-Ray Burst (sGRB). The sGRB, extensively studied by the NASA mission Swift (Gehrels, N. et al.2009), is believed to be due to a compact object merger. Analysis of a gravitational wave signal coincident with an EM signal from an sGRB will definitively determine whether the event is due to an NS-NS or NS-BH merger.

In the ISS telescope observational scenarios described next, we will consider first the known optical and X-ray afterglow signatures which follow the prompt gamma-ray signal of the sGRB. The sGRB afterglows decay with a several hour timescale, and are excellent candidates for follow-up detection within an ISS orbital period of 90 minutes. The engine for the extremely high energy sGRB has been established with a reasonable degree of confidence as an NSNS or NS-BH merger by examining the stellar populations associated with observed sGRBs (Bloom, J. et al. 2006); this implies a known gravitational wave signal associated with this source. We can estimate the rate of a GW/X-ray or GW/optical coincidence for the sGRB as follows. The observed sGRB rate (Guetta, D. \& Piran, T. 2006, Coward, D. et al. 2012) is $\sim 10 /\left(\mathrm{Gpc}^{3} \mathrm{yr}\right.$ ) (which includes beaming of the prompt gamma-ray into a nominal $15 \mathrm{deg}$ cone); within the advanced LIGO range of $200 \mathrm{Mpc}$ for a randomly oriented NS-NS merger, this gives an afterglow coincidence rate of $0.3 / \mathrm{yr}$, assuming the afterglow has the same beaming as the prompt signal. However two factors enhance this rate. First, the amplitude of a gravitational wave is peaked along the GRB axis by a factor of 1.5 . Second, the requirements of a time coincidence between the GW and prompt gamma-ray, and a spatial coincidence between the GW and the X-ray or optical afterglow source locations, can lower the GW network threshold by a factor of 1.5 while keeping the same false positive rate ${ }^{3}$. Together these two factors

\footnotetext{
3 The sensitivity improvement from requiring an EM coincidence depends on both the gravitational-wave background rejection factor, and the empirical shape of the background about the threshold of interest. The factor of 1.5 used in this calculation is consistent with a factor of $10^{7} \mathrm{GW}$ background rejection from the requirement of a prompt short gamma-ray coincidence within $5 \mathrm{sec}$ and X-ray afterglow
} 
will extend the gravitational wave detection range by 2.3 , leading to a coincidence rate of $4 / \mathrm{yr}$. For the NS-BH merger engine, the advanced LIGO range is $400 \mathrm{Mpc}$, and similar reasoning gives an EM/GW coincidence rate of 30/yr. These rates need to be adjusted for the efficiency of the X-ray and optical afterglow detection by the ISS telescopes, discussed below.

We also consider the signature for a so-called kilonova (Metzger, B. et al. 2010), a predicted (but not yet observed) optical afterglow from a NS-NS merger, powered by heating from the radioactive decay of heavy elements created in the merger ejecta. A significant advantage in the detectability of the kilonova coincidence with a gravitational wave is that its optical emission is believed to be isotropic, so that its rate is not limited by the possibility of beaming away from the earth (Metzger, B. \& Berger, E. 2012). Since all NS-NS mergers are expected to result in a kilonova, we use a NS-NS merger rate of 1,000/(Gpc ${ }^{3}$ yr) (Abadie, J. et al.2010) and a sky-average range of $200 \mathrm{Mpc}$ for randomly oriented NS-NS mergers to predict a GW-EM rate of 40/yr. (For the kilonova there is no preferred orientation, and the optical afterglow is expected to occur amidst a significant background level, and thus the GW range enhancements discussed above do not apply. Also, the difference in kilonova and sGRB rates of a factor of 100 can be reconciled through the beaming associated with the sGRB emission.) Finally, kilonova emission still occurs in low-density environments where sGRB optical afterglows become very weak.

\subsection{ISS telescope pointing}

In the event of a gravitational wave candidate signal from an NS-NS or NS-BH merger, an alert can be issued to the astronomical community within 5 minutes (Abadie, J. et al. 2012a). The alert will contain the sky localization error box associated with the event, and will be immediately available to the ISS telescopes through the continuous ISS data link. Both OpTIIX and ISS-Lobster will be mounted on pointing platforms that can slew to a given sky position, with a time lag dependent on the ISS position. The maximum delay for ISS-Lobster to access the LIGO-generated sky position will be slightly less than $1 / 2$ the period of the ISS orbit, or 40 min, with an average delay equal to half the maximum, or 20 min. OpTIIX is expected to be able to schedule followup observations within the next orbit, which makes it an ex-

within $100 \mathrm{deg}^{2}$, and a GW background distributed approximately exponentially with rate $\sim 100^{-\mathrm{SNR}}$ about $\mathrm{SNR}=10$ (e.g. the two-detector NS/NS background in Abadie, J. et al. (2012c), figure 3. The X-ray transient rate within $100 \mathrm{deg}^{2}$ was estimated from Greiner, J. et al. (2000), which looked for transients visible for 7.5 hours or less at a flux limit of $\sim 10^{-12} \mathrm{erg} / \mathrm{s} / \mathrm{cm}^{2}$. 32 potential candidates were found in a survey coverage of 76,000 deg 2 day, giving a rate of $\sim 10^{-4}$ transients per $\mathrm{deg}^{2}$. This may be extrapolated to $10^{-3}$ per $100 \mathrm{deg}^{2}$ at $10^{-11}$ $\mathrm{erg} / \mathrm{s} / \mathrm{cm}^{2}$ as a rough estimate for the Lobster transient rate.

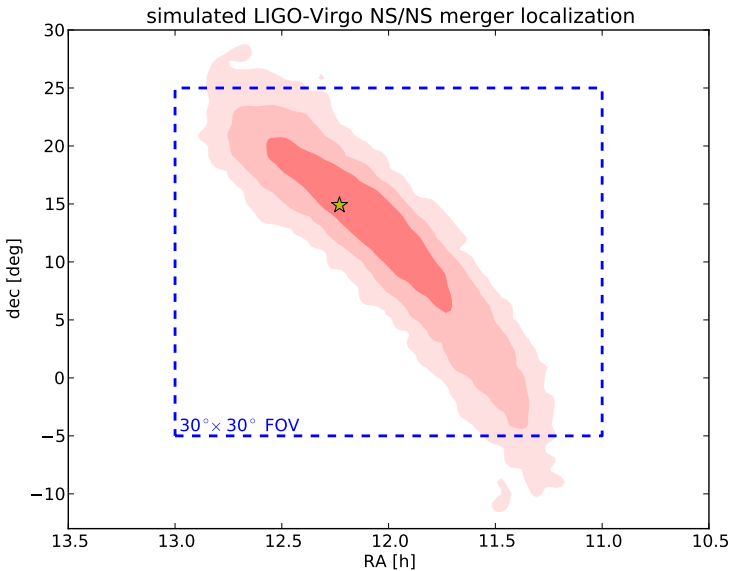

Fig. 5 ISS-Lobster FOV (dotted line) and NS-NS location error box (Veitch, J. \& Vecchio, A. 2010) where contours indicate 1, 2 and $3 \sigma$ confidence. The star indicates the true source location.

cellent complement to a related ground-based optical telescope follow-up effort (Kanner, J. et al. 2008; Abadie, J. et al. 2012b); a further advantage of the OpTIIX ISS deployment is the increased angular resolution possible above the atmosphere.

In order to maximize the likelihood of detecting the electromagnetic counterpart to a GW trigger, ISS-Lobster will begin pointed observation as soon as a GTM short duration trigger occurs by tiling the $2 \pi$ steradian GTM FOV with the WFI in 25 short $(\sim 1 \mathrm{~min})$ pointings starting from the zenith direction, until a source is either detected or the GW position is uploaded.

The main impediment to full sky viewing on the ISS are the solar panels, whose position varies on a daily and seasonal basis, and the Sun; after accounting for these factors, the average viewing efficiency of ISS-Lobster and OpTIIX are expected to be about $80 \%$. To avoid excessive repointings from the large number of false positives associated with LIGO sub-threshold triggers, a coincidence with the sGRB prompt gamma-ray signal, obtained with the ISSLobster NaI detector, will be required before either telescope is slewed to the LIGO sky position.

An important issue for the telescopes is locating the source within the $100 \mathrm{deg}^{2}$ gravitational wave error box. ISS-Lobster has a significant advantage here, as its large $30^{\circ} \times 30^{\circ}$ field of view allows it to encompass the gravitational wave sky error box from a NS-NS merger with a network SNR of 10 , as shown in figure 5, and localize the source to 1 arcmin. The large FOV for ISS-Lobster is optimal for the early phase of operation of the gravitational wave detector network ( 2017), with three detectors and a sky localization error box of $100 \mathrm{deg}^{2}$. In contrast, covering the full error box by tiling observations with OpTIIX would require an excessively large and impractical number of pointings, since its 


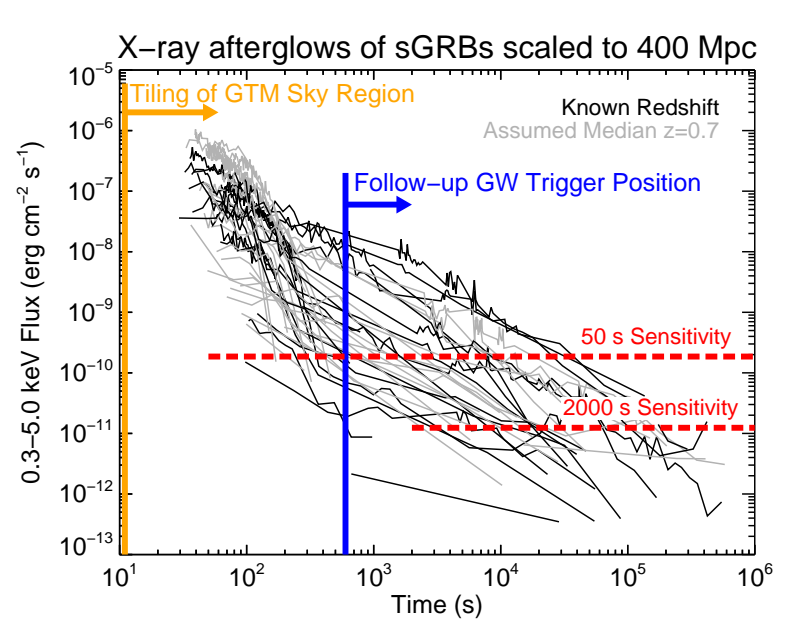

(a) Swift sGRB X-ray afterglows scaled to GW horizon (400 Mpc). The vertical lines represent the fastest response times for the follow-up scenarios. At least $80 \%$ of the afterglows will be detectable by ISS-Lobster.

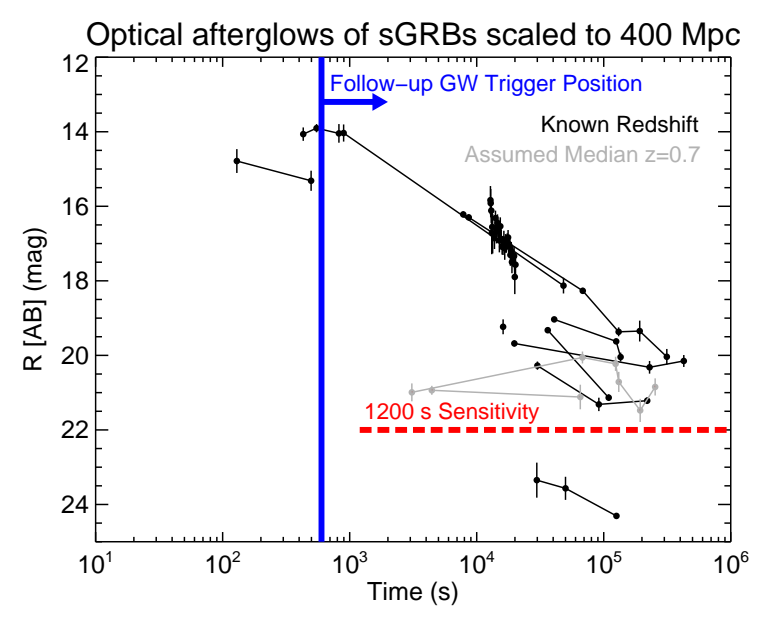

(b) sGRB optical afterglows with OpTIIX threshold

Fig. 6 Swift known-sGRB afterglow light-curves with flux re-scaled to $400 \mathrm{Mpc}$

FOV is only 3 arcmin. Instead, the detection of the optical afterglow of an sGRB could be enabled by first localizing the source position with ISS-Lobster to 1 arcmin, so that it can be contained within the OpTIIX field of view. In this case the OpTIIX detection rate is limited by ISS-Lobster.

If ISS-Lobster is not available, another possible solution, for sources within $100 \mathrm{Mpc}$, is to restrict OpTIIX pointings to galaxies within the LIGO range, since the location of a compact merger (as seen as an sGRB) appears to track the total mass of galaxies. For this purpose a galaxy catalog was assembled from publically available information, known as the GW Galaxy Catalog (GWGC) (White, D. et al. 2011). This catalog $(60 \%$ complete at present $)$, contains $\sim 50,000$ galaxies out to a distance of $100 \mathrm{Mpc}$, with roughly one galaxy per square degree of sky, or 100 hosts for a typical sky position error of $100 \mathrm{deg}^{2}$. By using a weighting with the galaxy luminosity (a proxy for total mass), the potential number of hosts can be further reduced to $\sim 40$, which captures $90 \%$ of the total catalog luminosity, and can finally be reduced to 10 , by requiring the capture of $50 \%$ of the total luminosity, resulting in $(0.6 \times 0.5)$ or $30 \%$ total probability of detection. This is a number of pointings ( 1 or 2 per orbit) that could be realistically accomplished by OpTIIX within the day-long decay time of an optical afterglow. An upgrade to GWGC to increase its listings, optimize its performance and increase its range is ongoing 4

\subsection{ISS-Lobster follow-ups}

At least $80 \%$ of sGRBs produce an X-ray afterglow, as detected by Swift within the first few hundred seconds. The efficiency of detecting an X-ray afterglow with ISS-Lobster is indicated in figure 6(a), where the flux from 43 sGRBs (17 with measured redshift, and 26 with no redshift) is plotted versus time, with the redshift used to scale the source flux to a distance of $400 \mathrm{Mpc}$ (those GRBs with no measured redshift are considered to be located at a median redshift of 0.7.) The vertical lines indicate the minimum times to begin WFI observation after a GTM sGRB trigger and subsequent tiling, or follow-up observations of GW trigger position. Roughly $80 \%$ of the afterglows will be detectable with the WFI. Including the ISS viewing efficiency (80\%), a conservative X-ray/GW coincidence rate is 1-2 sGRB/yr (at $440 \mathrm{Mpc}$; NS-NS) and 12 sGRB/yr (at $900 \mathrm{Mpc}$; NS-BH). These rates are also based on the assumption that the GW detector network is operating at full sensitivity (200 Mpc for LIGO, $100 \mathrm{Mpc}$ for Virgo) and 80\% duty cycle.

We note that these rates assume that the X-ray afterglow is beamed to the same degree as the prompt signal, however the evidence for this is weak at best, as evidence for beaming (an achromatic break in the X-ray light curve) is seen in only two or three of the 43 sGRB afterglows (Coward, D. et al. 2012). If the afterglow is more isotropic than the prompt signal, as some models assume (Metzger, B. et al.2008, Zhang 2012), the X-ray/GW coincidence rates will go up accordingly (in this case we may modify the prompt coincidence requirement with the GTM.)

\subsection{OpTIIX follow-ups}

About $30 \%$ of all sGRBs have optical afterglows. The optical afterglow to an sGRB within the LIGO detection range will appear bright (16-18 mag) at times less than 5 hours after the gravitational wave alert, and then dim significantly over the course of a day. Thus OpTIIX will be able to observe the decay of the light curve by repeated exposure to

\footnotetext{
4 J. Kanner, private communication
} 
the source over multiple ISS orbits. Figure 6(b) shows the flux from 13 sGRBs ( 8 with measured redshift, and 5 with no redshift) plotted versus time, with the redshift used to scale the source flux to a distance of $400 \mathrm{Mpc}$ (those GRBs with no measured redshift are considered to be located at a median redshift of 0.7.) The vertical dotted line is set at the average time required for a source to come in view by OpTIIX, and the horizontal line shows the sensitivity. All the afterglows are bright enough to be detected. Assuming OpTIIX is pointed to the source location by first imaging the $\mathrm{X}$ ray afterglow to 1 arcmin with ISS-Lobster, the sGRB opti$\mathrm{cal} / \mathrm{GW}$ coincidence rate is $1 / \mathrm{yr}$ for the afterglow of the NSNS merger engine, and 4/yr for the NS-BH merger. Again, these rates will increase if the afterglow beaming is more isotropic than the prompt gamma-ray.

The isotropic kilonova optical afterglow has a longer timescale than that of the sGRB. It is predicted to peak around 1 day after the NS-NS merger with a magnitude between 19 and 22, and decay over the following 3 days. Again assuming that the source is first localized by the (beamed) X-ray afterglow with ISS-Lobster, the OpTIIX detection rate for the kilonova optical/GW coincidence is $\sim 2 / \mathrm{yr}$. In contrast, if the source is within $100 \mathrm{Mpc}$, the galaxy map can be used to guide the OpTIIX search for the kilonova signal with about 10 pointings, yielding a rate of about $1 / \mathrm{yr}$ given the $30 \%$ detection probability achievable with the galaxy catalog mentioned above. This may be an important followup source in the time period before LIGO reaches its full sensitivity. Finally, addressing the difficult problem of distinguishing a kilonova from background transient events including supernovae and variable stars will require an accurate measure of the candidate signal time variance, measured over successive ISS orbits. The ISS deployment of OpTIIX will mean that atmospheric fluctuations will not be present in the measurement of source variability (a key challenge for groundbased optical telescopes).

\section{Conclusions}

The ISS platform offers a unique and inexpensive opportunity for electromagnetic follow-up studies of gravitational wave sources. These follow-up studies will offer a wealth of complementary science including determining the source location and redshift, removing degeneracies in the GW analysis, and raising the signal-to-noise of the event. The technology demonstrator OpTIIX, a 1.5 m diffraction limited optical telescope, is scheduled for ISS operation in 2016. ISSLobster, a time-domain X-ray observatory, is in the conceptual design phase for a proposal to a Mission of Opportunity, which could be operational in 2017. These schedules coincide well with the planned operation of the LIGO and Virgo gravitational wave detectors.
Both OpTIIX and ISS-Lobster will be mounted on pointing platforms in continuous communication with groundbased gravitational wave alerts, allowing the fast acquisition of the source sky location. The source location within the large GW sky error box $\left(\sim 100 \mathrm{deg}^{2}\right)$ expected in the initial operating phase of the GW network can be imaged within the FOV of ISS-Lobster to 1 arcmin, allowing a follow-up by OpTIIX and localization to 1 arcsec. Use of a galaxy catalog can also independently guide the pointing of OpTIIX if the source is within $100 \mathrm{Mpc}$.

With the assumptions that 1) a short GRB is powered by either an NS-NS or an NS-BH merger, 2) the X-ray and optical afterglows of an sGRB are beamed into the same opening angle as the prompt gamma-ray, and 3) a kilonova is the correct description of the long-term optical afterglow of a NS-NS merger, the coincident rates for EM/GW signals are: X-ray signal from sGRB with NS-NS (NS-BH) merger engine, 2/yr (12/yr); optical signal from sGRB with NS-NS (NS-BH) merger engine, 1/yr (4/yr); optical signal from NSNS kilonova using galaxy catalog to guide pointing, 1/yr; optical signal from NS-NS kilonova using ISS-Lobster to localize source, $2 / y r$. These rates encourage the planned use of these ISS telescopes for electromagnetic follow-up to gravitational wave detections, an exciting emerging astronomical field which is certain to contain sources and surprises beyond those described here.

\section{References}

Abadie, J. (LIGO Scientific Collaboration) 2010, CQG, 27, 173001

Abadie, J. (LIGO Scientific Collaboration) 2012, A\&A , 541

Abadie, J. (LIGO Scientific Collaboration) 2012, A\&A, 539, A124

Abadie, J. (LIGO Scientific Collaboration) 2012, Phys. Rev. D 85, 082002

Abbott, B. (LIGO Scientific Collaboration) 2009, Reports on Progress in Physics, 72, 076901

Abbott, B. (LIGO Scientific Collaboration) 2004, NIM A, 517, 154

Accadia, T. 2011, CQG, 28, 114002

Aurière, M. 1982, A\&A , 109, 301

Barthelmy, S., Barbier, L., Cummings, J. et al. 2005, Space Sci. Rev. $120,143-164$

Black, J., Brunton, A., Bannister, N. et al. 2003, NIM A, 513, 123

Bloom, J., Prochaska, J., Pooley, D. et al. 2006, ApJ , 638, 354

Coward, D., Howell, E., Piran, T. et al. 2012, MNRAS , 425, 1365

Dalal, N., Holz, D. \& Hughes, S. 2006, Phys. Rev. D , 74, 063006

Fairhurst, S. 2011, CQG, 28, 105021

Gehrels, N., Riuz, E. \& Fox, D. 2009, ARA\&A , 47, 567

Greiner, J., Hartmann, D., Voges, W. et al. 2000, A\&A , 353, 998

Grote, H. \& LIGO Scientific Collaboration 2010, CQG, 27, 084003

Guetta, D. \& Piran, T. 2006, A\&A , 453, 823

Harry, G. (for the LIGO Scientific Collaboration) 2010, CQG, 27, 084006

Hughes, S. \& Holz, D. 2003, CQG, 20, S65

Kanner, J., Huard, T., Marka, S. et al. 2008, CQG, 25, 184034

Leonhardt, V. \& Camp, J. 2006, Space Telescopes and Instrumentation I, Proceedings of SPIE, 6265, pp. 62652M

Matsuoka, M., Suzuki, M., Kawasaki, K. et al. 2010, Proceedings of X-ray Astronomy 2009, AIP Conference Proceedings, 1248, 531536 
Metzger, B., Penendo, G., Darbha, S. et al. 2010, MNRAS , 406, 2650 Metzger, B. \& Berger, E. 2012, ApJ , 746, 48

Metzger, B., Quataert, E. \& Thompson, T. 2008, MNRAS , 385, 1455

Numata, K., Camp, J., Krainak, M. et al. 2010, Optics Express, 18, 22781

Postman, M., Sparks, W., Liu, F. et al. 2012, Proceedings of SPIE, $8442-63$, in press

Sathyaprakash, B., Fairhurst, S., Schutz, B. et al. 2012, LIGO Document T1200219

Somiya, K. (for the KAGRA collaboration) 2012, CQG, 29, 124007

Veitch, J. \& Vecchio, A. 2010, Phys. Rev. D , 81, 062003

White, D., Daw, E. \& Dhillon, V. 2011, CQG, 28, 085016

Zhao, F., Rao, S., Ksenszov, A. et al. 2006, NASA technical report, http://hdl.handle.net/2014/40796

Zhang, B. 2012, arXiv:1212.0773 